in den Zellen und ihre Verwertung für die Frage nach der Herkunft des Fettes. Verhandlungen der dentschen pathologischen Gesellschaft, sechste Tagung.

4. Albrecht, Über trübe Schwellung und Fettdegeneration. Verhandlungen der dentschen pathologischen Gesellschaft, sechste Tagung.

5. Schwalbe, Uber Fettwanderung bei Phosphorvergiftung. Verhandlungen der deutschen pathol. Gesellschaft, sechste Tagung.

6. Rosenfeld, Fragen der Fettbildung. Verhandlungen der deutschen pathol. Gesellschaft, sechste Tagung.

7. Dietrich, Wandlungen von der. Lehre der fettigen Degeneration. Arbeiten aus dem pathol.-anat. Institut zu Tübingen, Bd. V, Heft 1. 1904.

8. Pio Foa (Turin), Beitrag zur Kenntnis der Fettinfiltration. Verhandlungen der deutsch. pathol. Gesellschaft, achte Tagung.

9. Hans Wuttig, Experimentale Untersuchungen über Fettaufnahme und Fettablagerung. Zieglers Beiträge, Bd. 37, Heft 2.

10. Hagemeister, Beiträge zur Kenntnis des Fettschwundes und der Fettbildung in ihrer Abhängigkeit von Circulationsveränderungen. Dieses Archiv, Bd. 172, 1903.

11. Arnold, Die Bedeutung der Fettsynthese, Fettphagocytose, Fettsekretion und Fettdegeneration für die Milch- und Kolostrumbildung. Münchn. Med. Wochenschr. Nr. 18, 2. Mai 1905.

\title{
XXII.
}

\section{Über die Entstehung der Amyloidkörperchen im Zentralnervensystem.}

(Aus dem Laboratorium der Psychiatrischen Klinik zu Florenz.)

\author{
Von \\ Dr. G. Catola, \\ Assistenten in der Psychiatrischen Klinik \\ und \\ N. Achúcarro, \\ Arzt (Bilbao). \\ (Hierzu Tafel XII und 2 Textfiguren.)
}

Die Amyloidkörperchen stellen einen häufigen Befund im Zentralnervensystem dar. Deshalb ist die Frage ihrer Entstehung wichtig. Obwohl sie vielfach behandelt worden ist, sind die Meinungen der Autoren sehr geteilt, wie aus folgendem 
kurzen, nur das Wichtigste berücksichtigenden Überblick leicht zu sehen ist.

Purkinje ${ }^{1}$ war der erste (1837), der diese Körperchen im Zentralnervensystem fand.

Virchow ${ }^{2}$ beschrieb sie (1854) im Gehirn und im Rückenmark unter dem Namen von Cellulosekörperchen und als kleine Massen, die nach ihm von ähnlicher Zusammensetzung waren, wie die pflanzliche Cellulose und dadurch von den als Gehirnsand bekannten Körperchen verschieden. Zuerst fand sie Virchow nur in der Nähe des Ependyms und meinte, nur wo Ependym sei, könnten sich Cellulosekörperchen bilden.

Ähnliche Gebilde waren schon (1723) von Morgagni mit dem Namen von Corpora Amylacea in der Prostata beschrieben worden.

Luschka ${ }^{3}$ fand sie im Ganglion Gasseri (1854-55).

Virchow ${ }^{4}$ beschrieb sie noch (1855) in großer Zahl in den Erweichungen des Gehirns und des Rückenmarks und auch im Plexus choroideus. Er betrachtete diese Körperchen als von derselben Natur wie die in der Prostata beschriebenen. Über die Entstehung solcher Körperchen im Nervensystem dachte Virch ow (1871), daß dieselbe ein sekretorischer Vorgang wäre.

Leber ${ }^{5}$ (1875) läßt die Amyloidkörperchen, die er im Rückenmark und im Opticus fand, aus den Nerrenfasern entstehen. Treite $1^{6}$ unterstützte 1876 diese Meinung.

Leyden ${ }^{7}$ fand sie (1880) in der grauen Degeneration des Rückenmarks und besonders auch bei Senilen.

$\mathrm{Ceci}^{8}$ läßt (1881) die Amyloidkörperchen aus der Gerinnung und Umbildung des Nervenmarkes entspringen.

Klebs meinte 1889, daß aus den Gliazellen die Amyloidkörperchen sich bildeten.

Schaffer ${ }^{10}$ untersuchte 1890 die Amyloidkörperchen im Rückenmark von an Lyssa gestorbenen Individuen und glaubte, daß der Vorgang der Entstehung der betreffenden Körper in einer chemischen Umwandlung des Achsenzylinders und der Markseheide $z u$ finden sei, die mit dem Verlust der optischen Differenz zwischen beiden Bestandteilen und deren Zusammenschmelzung endete. 
Redlich ${ }^{11}$ glaubt (1891), daß die Amyloidkörperchen aus gewissen Veränderungen der Gliazellen entstehen.

Siegert ${ }^{12}$ betrachtet (1892) die Amyloidkörperchen als Kombinationsprodukte des degenerierten Markes mit der Lymphe des umgebenden Gewebes.

Wichmann ${ }^{13}$ hält (1893) die Substanz, die die Amyloidkörperchen bildet, für aus den Gefäßen stammend. Sie würde unter normalen Verhältnissen im Stoffwechsel des Nervengewebes verbraucht werden und nur unter pathologischen Bedingungen nicht mehr von den Nervenelementen aufgebraucht werden und im Gewebe als Amyloidkörperchen sich niedersetzen.

Stroebe ${ }^{14}$ fand 1894 nach gänzlicher oder teilweiser Durchschneidung des Rückenmarks von Kaninchen gewisse kugelige Gebilde, die er als Amyloidkörperchen erklärte. Er beschrieb die Entstehung dieser Gebilde aus der Degeneration der Achsenzylinder unter Beteiligung der Markscheide. Wenige Tage nach der Durchschneidung des Rückenmarks zeigten die Achsenzylinder kugelige oder birnförmige Anschwellangen, aus welchen die freien kugeligen Gebilde entstanden.

Obersteiner ${ }^{18}$ u. 21 (1900 und 1903) rerteidigt die Ansicht, daß gewisse eigentümliche, hellglänzende Gebilde, die mit Osmiumsäurefärbung einen schwarzen Rand zeigten und sich in den Gliazellen des senilen Gehirns fanden, das Vorstadium in der Bildung der Amyloidkörperchen darstellten.

Wolf ${ }^{19}$, ein Schüler von Schmaus, hält 1901 die Amyloidkörperchen für Degenerationsprodukte der Nervenfasern. Sie entstehen allmählich ohne weitere Formveränderung nur mit Änderung ihrer chemischen Zusammensetzung aus den Myelintropfen, die wieder direkt aus Nervenfasern entstehen und sich meist aus Achsenzylinder- und Markscheideteilen zusammensetzen.

In der letzten Zeit haben wir einige Fälle aus dem Laboratorium von Prof. Marie in Bicêtre und aus der Psychiatrischen Klinik von Florenz untersuchen können, bei welchen die Amyloidkörperchen ungewöhnlich zahlreich waren.

Die Fälle, die wir besonders benutzt haben, sind zwei von Paralysis Agitans, zwei von Syringomyelie und drei von 
seniler Demenz. Die von den Amyloidkörperchen bevorzugten Stellen des Nervensystems, nämlich die Randzone und die hinteren Stränge des Rückenmarks, die Medulla oblongata, die Gegenden um den Hirnventrikeln und besonders die Stria cornea und den Bulbus olfactorius sind näher berücksichtigt worden.

Für die Untersuchung haben wir folgende Färbungsmethoden angewandt: Hämatoxylin-Eosin, Toluidinblan, Nigrosin, Weigertsche Markfasernfärbung, Weigert-P alsche Färbung mit Cochenille oder Safranin als Kontrastfärbung, die Methode von Stroebe für Achsenzylinder, van Gieson, Bordeaux, Fixierung in Alkohol mit Salpetersärre, Färbung mit Toluidinblau und Fixierung der Farbe durch Ammonmolybdän nach L ug a ro, Silberimprägnation und Reduktion mit Formol-Pyrogallussäure nach Cajal (Fixierung in ammoniakalischem Alkohol), Methode von Kaplan für Achsenzylinder (Anthraceneisengallustinte, Differenz. nach Pal, Kontrastfärbung mit Fuchsin). Dazu wurde das Jod als Identifizierungsmittel für Amyloidkörperchen gebraucht.

Außer den jetzt näher untersuchten Fällen haben wir frühere Präparate von verschiedenen Krankheiten des Nervensystems nachgesehen, bei welchen Amyloidkörperchen zu sehen waren. Dies waren Fälle von Hemiplegie, von Paralyse, von Sclérose combinée, von Sclerosis multiplex, von Tabes usw. Diese Präparate waren hauptsächlich mit der Weigertschen Markfärbung behandelt worden und deswegen nicht für unsere Untersuchung im Sinne der Entstehung der Amyloidkörperchen sehr geeignet. Doch vermochten sie über die Verteilung der Körper im Zentralnervensystem Auskunft zu geben. Wir konnten konstatieren, dab in der Tat die Amyloidkörperchen besonders zahlreich in den hinteren Strängen und in der Peripherie des Rückenmarks, an den Hirnventrikeln und im Bulbus olfactorius hervortreten. Im allgemeinen sind sie um die Gefäße stärker angehäuft.

Von allen den erwähnten Methoden sind es diejenigen von van Gieson, Stroebe, Cajal und die von Kaplan, welche für die Erklärung der Entstehungsweise der Amyloidkörperchen die geeignetsten Resultate geliefert haben. Im Einklang mit den Vermutungen, die diese Arbeit veranlaßst haben, sind dio Methoden dor Achsenzylinderfärbung hier am besten zu verwerten, denn unsere Untersuchungen haben 
bestätigt, daß die Amyloidkörperchen des Nervensystems als Degenerationsprodukte der Achsenzylinder aufzufassen sind. Die günstigsten Fälle unter denen, die wir untersucht haben, waren einer von Paralysis agitans und einer von Syringomyelie, besonders der von Paralysis agitans. Im Rückenmark waren in diesem Falle die Amyloidkörperchen äußerst zahlreich verbreitet und doch nicht zu dicht angehäuft, so daß neben ganz zerstörten Fasern andere von normalem Ansehen und solche, die wir als Utbergangsformen betrachten, zu sehen waren.

Daß die Amyloidkörperchen Degenerationsprodukte der Nervenfasern sind, haben Leber, Treitel, Schaffer, Stroebe, Wolf geäußert. Diese Ansicht ist aber in der letzten Zeit von Obersteiner scharf bekämpft worden. Obersteiner sagt: „Es ist mir unbegreiflich, wie eine so irrige Auffassung von geübten Mikroskopikern propagiert werden konnte, schon Längsschnitte durch das Rückenmark müßten ja jeden belehren. " Die Längsschnitte durch das Rückenmark haben sich bei unserer Untersuchung als sehr lehrreich erwiesen, aber im entgegengesetzten Sinne als Obersteiner ausführte. Demnach werden wir unsere Beschreibung besonders auf frontale Schnitte des Rückenmarks im Gebiet der hinteren Stränge stützen.

Die Amyloidkörperchen treten hier als kugelige Gebilde, von einer Größe von 18 bis $25 \mu$ auf. Die kugelige Form ist bei weitem die häufigste, doch trifft man solche Gebilde, die oval oder länglich sind und zuweilen, wenn auch äußerst selten, sieht man solche, die fast eine polygonale Figur zeigen. Die Größe der Amyloidkörperchen eines und desselben Falles ist gewöhnlich ziemlich gleichmäßig, doch findet man kugelige Körperchen, die riesige Dimensionen haben im Vergleich zu den anderen. So können sie die doppelte Größe eines gewöhnlichen Amyloidkörperchens erreichen. In Fig. 1, Taf. XII sieht man zwei Amyloidkörperchen, die in enger Verbindung stehen. Solche Bilder sind nicht sehr selten and wir würden geneigt sein, sie vielmehr als eine Zusammenschmelzung, denn als eine Teilung darstellend zu betrachten. Auf diese Weise würde die Anwesenheit solcher riesiger Körperchen leicht erklärlich sein. 
Was die Färbung der Amyloidkörperchen betrifft, so ist sie von unsicherem Charakter. Außer ihrer Färbung durch das Jod, das immer noch das beste Mittel zur Identifizierung der Amyloidkörperchen darstellt, ist das weitere Verhalten der Färbungsmittel dagegen wenig elektiv. Fast alle angewandten Färbungsmittel färben die Körperchen tief progressiv. Die Farbe geht aber leicht verloren, wenn man regressive Methoden anwendet. Diese leichte und wenig elektive Färbbarkeit der Amyloidkörperchen gibt den Eindruck, als ob die chemischen Affinitäten dieser Körper zu den Färbemittel gering sind und die Färbung nur Resultat einer physikalischen Imprägnation ist. Bei der Silberimprägnation nach Cajal bleiben die Körperchen jedoch hellgelb gefärbt, während die Achsenzylinder ganz schwarz oder auf jeden Fall viel tiefer gefärbt erscheinen.

Für das Hämotoxylin haben aber diese Körperchen eine gewisse Vorliebe, doch nicht alle zeigen diese Eigenschaft in demselben Grade. Kölpin will in diesem Umstand ein sicheres Erkennungsmittel für die betreffenden Körperchen sehen, womit man sie leicht von den gequollenen Achsenzylindern unterscheiden soll. Nach ihm, wenn man die Färbungsmethode von van Gieson anwendet, färben sich die Achsenzylinder rötlich-violett, während die Amyloidkörperchen tief blau werden. In unserem Falle von Paralysis agitans haben wir die Methode nach van Gieson gebraucht, und wir haben eine große Buntheit der Amyloidkörperchen bemerkt. Die Mehrzahl war blau gefärbt, andere jedoch tiefrot und welche mit gemischten Farbentönen.

Daß alle diese Körper derselben Natur waren, ist nicht zu bezweifeln, denn die Verwechslung mit Achsenzylindern im Längsschnitte ist leicht $\mathrm{zu}$ vermeiden und außerdem waren alle Körperchen in anderen Schnitten mit Jod gefärbt. Diese Erscheinung mag zeigen, daß die von Kölpin geschätzte Erkennungsweise nicht zuverlässig genug ist. Diese Buntheit der Färbung ist bei allen doppelten Methoden zu konstatieren. $\mathrm{Daß}$ die Amyloidkörperchen eine tief färbbare zentrale Zone zeigen, haben viele Autoren beschrieben. Die Deutung dieser Erscheinung ist aber von verschiedenen Seiten eine andere gewesen. Während einige nur von einer Schichtung der 
Substanz der Amyloidkörperchen sprechen, halten andere dieselben als aus zwei chemisch und physikalisch verschiedenen Teilen bestehend. Durch regressive Färbung mit Kontrastnachfärbung bekommt man solche Bilder wie sie in unseren Figuren (die Präparate nach Kaplan abbildend) dargestellt worden sind. Diese Bilder scheinen uns aber Kunstprodukte $\mathrm{zu}$ sein, die von dem Grade der Differenzierung abhängig sind, und die den Bildern, die Fischer bei der Doppelfärbung von künstlich aus Albuminoiden gefällten Granula erlangt hat, ganz entsprechen. So sagt Fischer ${ }^{17}$ : "Wie bei einer Schützenscheibe tritt ein allein noch gefärbtes Zentrum oder ein Spiegel scharf hervor, umgeben von einem ganz entfärbten oder durch Kontrastfärbung secundär färbbaren Rand oder Schale oder Rahmen. Von dem Grade der Differenzierung hängt die Breite des Spiegels $a b$; er ist sehr breit bei geringer, sehr klein und einem Scheibenspiegel um so ähnlicher, bei stärkerer Differenzierung. Die Spiegeldifferenzierung ist weiter nichts als eine zur rechten Zeit unterbrochene Entfärbung der durchweg gleichartig gebauten Granula."

Die Erfahrungen mit den Amyloidkörperchen stimmen vollständig zu dieser Beschreibung. Auch bei unseren Körperchen ist die Größe des Zentrums im Vergleich zu dem umgebenden Rande nur von dem Grade der Differenzierung abhängig. Diese Erscheinung wird so bedingt und nicht etwa durch chemisch verschiedene Zonen der Amyloidkörperchen.

Anders zu deuten ist die konzentrische Schichtung und die körnige Beschaffenheit des Zentrums bei Anwendung einer einfachen Färbung. Diese so erzielten Erscheinungen mögen wohl auf wirklichen Strukturen der Amyloidkörperchen beruhen. $\mathrm{DaB}$ die vielfach erwähnten radiären Streifungen nur künstliche Sprünge, die besonders durch. Druck des Präparates hervorgebracht werden können, darstellen, wie angenommen wird, dem stimmen wir vollständig bei. Es bleiben also nur die konzentrische Schichtung und die körnige zentrale Beschaffenheit als Strukturen der Amyloidkörperchen. Ihr Auftreten ist aber selten, und in unseren Fällen war die Mehrzahl der Amyloidkörperchen homogen. Diese Strukturverschiedenheiten und vielleicht auch die kleinen Verschiedenheiten in der Färbung 
könnten als verschiedene Stadien eines und desselben Vorganges angenommen werden. Die konzentrische Schichtung, die sich nur bei Amyloidkörperchen zeigt, die frei im Gewebe sind, würde eine spätere Phase darstellen. Wir wollen aber jetzt die ersten Phasen, bevor die Amyloidkörperchen frei werden, berücksichtigen. Längsschnitte durch das Rückenmark zeigen neben normal gebliebenen Fasern andere, die dem Degenerationsvorgang fast vollständig anheimgefallen sind. Die normalen Fasern behalten ihre scharfen Grenzen und ihre spezifische Färbbarkeit, aber man findet neben diesen andere, welche unregelmäßig geworden sind und mehr oder weniger große Anschwellungen zeigen. Es gibt noch eine andere Art von Achsenzylindern, die, obwohl sich in ihren morphologischen Eigenschaften keine Veränderungen zeigen, doch in ihrer Färbbarkeit verändert sind. Die Färbbarkeit war derart verändert, daß bei der Stroebeschen Färbung auf blau gefärbte Strecken andere folgten, die durch Saffranin rot gefärbt waren. Die Abgrenzung beider Arten von Segmenten war eine ganz scharfe und die Länge der Segmente sehr verschieden. Mit. der Methode von Kaplan war ein ähnliches Bild zu sehen. Mit der Methode von Cajal wurde eine so scharfe Darstellung dieser Erscheinung erreicht, daß wir sie nicht mehr als einen Fehler der Methodik annehmen konnten. Hier folgten auf ganz schwarze Strecken andere, die einen gelblichrötlichen Ton hatten. Die Abgrenzung zwischen beiden Arten von Segmenten war ebenso scharf. Die schwarzen Segmente waren kürzer als die gelblichen, sodaß sie auch bei flüchtiger Beobachtung den Anschein der Faserneinschnürungen geben konnten, denn, wie bekannt, sind diese im Zentralnervensystem länger und folgen häufiger aufeinander, als in den peripherischen Nerven. Die Mikrophotographie 1 wurde aus einem solchen Achsenzylinder hergestellt. Wir haben in unseren Präparaten viele Stellen, die sich jedoch für die Mikrophotographie nicht eigneten, bei welchen die schwarzen Segmente viel näher aneinander an einem Achsenzylinder stehen, so daß sie unmöglich als physiologische Faserneinsclinürungen erkannt werden konnten. Wir glauben aber, daß der Grund beider Erscheinungen derselbe ist, nämlich der Mangel der 
Myelinscheide an bestimmten Stellen des Achsenzylinders. Die bei der Stroebeschen Methode rot gefärbten Segmente würden denjenigen, die bei der Silberimprägnation schwarz erscheinen, entsprechen. Bei den ersten würde durch den Mangel an Myelin eine leichtere Differenzierung stattfinden, bei den letzteren die Silberimprägnation und die Wirksamkeit der Reduktion leichter sein. In jedem Falle handelt es sich vielmehr um eine grobe physikalische Veränderung, als um eine Änderung der Färbbarkeit der Achsenzylinder. Diese Eigentümlichkeit ist hier nur als eine solche erwähnt, und es ist kein Grund, diese Erscheinung dem Vorgang der Amyloidkörperchenbildung zuzuschreiben.

Andere Achsenzylinder zeigten deutliche kleinere oder größere Anschwellungen von ovaler, spindelförmiger und kugeliger Form. Bei allen Färbungen traten sie deutlich hervor und immer bleicher gefärbt als die anderen Stellen der Achsenzylinder. Mit der Methode von Cajal sind sie hellgelb gefärbt.

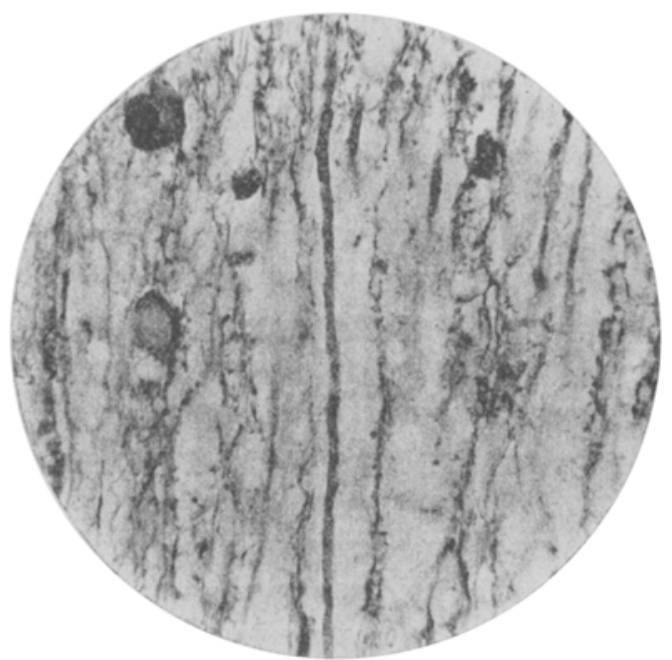

Fig. 1.

Mit der Färbung nach Stroebe erscheinen sie hellblau oder rötlich, und man sieht viele, die im zentralen Teil rötlich gefärbt sind, während die äußere Zone sich blau färbt und 
an dem Achsenzylinder fortsetzt. Es scheint, daß diese Veränderung ganz im zentralen Teil des Achsenzylinders stattfindet. Welchen Anteil die Myelinscheide bei diesem Vorgang nimmt, ist schwer zu entscheiden. Die Autoren, die die Ansicht der

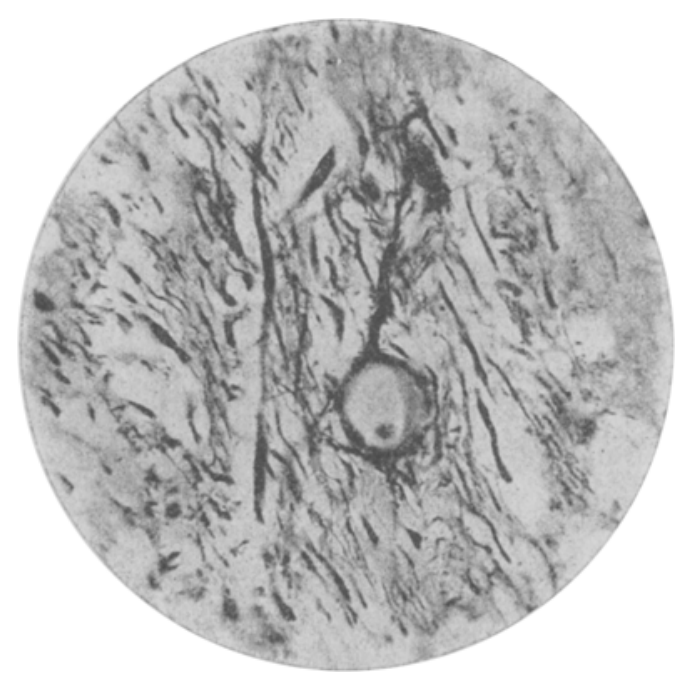

Fig. 2.

Entstehung der Amyloidkörperchen aus den Fasern verteidigen, lassen die Myelinscheide eine ebenso wichtige Rolle spielen, als den Achsenzylindern. Schaffer denkt an eine "Amalgamierung" der Substanzen des Achsenzylinders und der Markscheide. Wolf hält die Amyloidkörperchen für umgewandelte Myelintropfen.

Neben dieser Phase der Anschwellungen der Achsenzylinder ist eine andere $\mathrm{zu}$ finden, wenn auch selten, die die Grundphase für die Erklärung des Vorganges darstellt, nämlich diejenige, wo ein ausgebildetes Amyloidkörperchen von ganz gleicher Beschaffenheit wie die derjenigen, die man frei im Gewebe findet, in direkt anatomischer Abhängigkeit von einem Achsenzylinder steht. Diese Bilder sind etwas selten, denn, wie schon Stroebe bemerkt, die Achsenzylinder verlieren bald ihre Färbbarkeit, sie zerstückeln sich, und ihr Zusammenhang 
mit den Amyloidkörperchen ist schwer zu sehen. Die Mikrophotographie 2 stellt ein solehes Amyloidkörperchen in $\mathrm{Zu}$ sammenhang mit einem Achsenzylinder dar. Auch unsere Figuren 2 and 3 enthalten Beispiele solcher Formen.

Die Amyloiḍörperchen werden später aus ihrem Zusammenhang mit dem Achsenzylinder ganz gelöst und man sieht oft Kugeln, die noch gewisse Zeichen ihrer früheren Abhängigkeit von dem Achsenzylinder behalten haben. Es sind kleine, tief gefärbte Klümpchen, die aus der Zerstörung der Achsenzylinder stammen und die dem Amyloidkörperchen an einer Stelle oder an zwei gegenüberstehenden Stellen anhaften. Die befreiten Amyloidkörperchen behalten ihre Längsanordnung und bilden auf diese Weise lange Ketten, zu denen jetzt ein neues Element, nämlich die Gliazellen, hinzutritt. Es sind Zellen, die gewöhnlich einen hellgefärbten Kern zeigen und manchmal ein breites, körniges Protoplasma. Diese Zellen stellen sich zwischen die Amyloidkörperchen in den erwähnten Ketten. Diese Zellen passen sich der konvexen Fläche der Amyloidkörperchen, mit denen sie in Berührung kommen, ganz an, und die Körperchen erscheinen manchmal teilweise in der konkaven Fläche der Zellen eingeschlossen. Wäre diese Erscheinung so isoliert zu sehen, so könnte man der Meinung Obersteiners, nämlich daß die Amyloidkörperchen Produkte einer Veränderung des Gliaprotoplasmas sind, zustimmen. Übergangsformen nach dieser Richtung haben wir nicht finden können, und wir sind deshalb überzengt, daß es sich hier um eine secundäre Erscheinung handelt, die die Amyloidkörperchen als Fremdkörper seitens der Glia hervorrufen. Was aus den Amyloidkörperchen in späteren Stadien wird und wie sie von den Gliazellen beeinflußt sind, ist uns völlig unbekannt. Man findet aber im Gewebe sehr oft Lücken, die unverkennbarerweise den Amyloidkörperchen entsprechen. Andererseits findet man Anhäufungen von solchen Körpern neben den Gefäßen, neben den Ventrikeln und an der Oberfläche des Gehirns und des Rückenmarks. $\mathrm{Ob}$ diese Stellen diejenigen der Entstehung der dort gefundenen Amyloidkörperchen 'sind, oder ob diese später, nach ihrer Bildung in den erwähnten Stellen, sich anhäufen, konnten wir nicht entscheiden. Die Tatsache ist von Obersteiner zugunsten 
seiner Ansicht über die Amyloidkörperchenentstehung gedeutet worden. Da die Amyloidkörperchen nach (Obersteiner) aus den Gliazellen entstehen, sollen sie sich besonders da finden, wo die Glia reichlich ist und wo die Achsenzylinder wenig zahlreich sind oder fehlen. Daß dies nicht unbedingt zutreffen muß, zeigt unser Fall von Syringomyelie. Hier waren die Amyloidkörperchen sehr zahlreich, und ihre Lage war mit der Gliaausbildung nicht zusammenfallend. Im Gegenteil, die große zentrale Höhle war von einer dichten perikavitären Gliaschicht umgeben, in welcher ganz ausnahmsweise Amyloidkörperchen zи sehen waren. Dagegen an den Rückenmarksträngen, wo die Glia die gewöhnliche Ausbreitung hatte, waren die Körperehen sehr zahlreich. Wir glauben demnach, daß die von Obersteiner als Stütze seiner Ansicht hergebrachte Tatsache keinen großen Beweis liefert. Die Möglichkeit ist aber nicht ausgeschlossen, daß die Amyloidkörperchen ein allgemeineres Degenerationsprodukt sind, so daß sie sowohl von den Achsenzylindern, wie auch von den Gliazellen ihren Ursprung entnehmen können. Um das letzte durch histologische Untersuchungen zu beweisen, müßte man so viele oder so ausdrucksvolle Übergangsformen finden, daß diese Erklärung sich von selbst ergibt.

Redlich, der die Entstehung der Körperchen aus den Gliakernen für wahrscheinlich hält, stützt seine Meinung auf folgende Gründe: auf die Vorliebe der Amyloidkörperchen für die nucleäre Farbe, auf die Anwesenheit eines Kerns in den Amyloidkörperchen in manchen Fällen, auf die Ähnlichkeit ihrer Lage im Gewebe mit derjenigen der Gliakerne und auf ihr vorzugsweises Erscheinen in den gliareichen und faserarmen Zonen des Nervensystems. Daß die Amyloidkörperchen einen gewissen Vorzug für nucleäre Farben zeigen, kann wohl nicht als Grund einer Entstehung aus den Kernen angenommen werden. Wenn die Amyloidkörperchen tatsäehlich aus Gliakernen entständen, so würden sie als eine Art Entartung der Kerne aufzufassen sein, die wahrscheinlich Veränderungen in der chémischen und grobphysikalischen Struktur und deswegen in der Färbbarkeit mit sich bringen würde.

Aus der Ähnlichkeit der Färbbarkeit eines Gewebselementes mit der eines Degenerationsproduktes kann man nicht die 
Entstehung des letzten aus dem ersten beweisen. In unseren Fällen sind bei Rückenmarklängsschnitten Amyloidkörperchen zu finden, die in unmittelbarem Zusammenhang mit Achsenzylindern stehen und deshalb als Anschwellungen und Veränderungen derselben aufzufassen sind und doch zeigen sie eine andere Färbbarkeit als die Achsenzylinder.

Was die Anwesenheit eines Kernes betrifft, so wissen wir schon, daß der Kern der Amyloidkörperchen nicht als ein Zellkern betrachtet werden kann.

Die Angaben Stroebes sind sehr wichtig, denn sie stellen neben den von Homen, welcher auch Amyloidkörperchen bei Durchschneidung des Rückenmarks fand, die einzigen Experimentalarbeiten auf diesem Gebiete dar. Die Abbildungen von Stroebe entsprechen im ganzen den Bildern, die wir mit seiner Methode erlangt haben. Doch der Vorgang muß in beiden Fällen etwas verschieden sein. Bei Betrachtung unserer Präparate sind wir zu der Ansicht gekommen, daß die Phase der Homogenisierung, die Stroebe bei der Bildung der Amyloidkörperchen in seinen Versuchstieren fand, in unseren Fällen nicht stattfindet. Die Anschwellungen der Achsenzylinder sind in unseren Fällen von Anfang an homogen und ebenso die daraus entwickelten Körperchen. Die konzentrische Schichtung oder die körnige Beschaffenheit der zentralen Zone kommen erst später. Die von Stroebe beschriebene Homogenisierung würde durch die in der Myelinscheide enthaltenen Substanzen sich vollziehen. Wir haben schon gesehen, daß auch Schaffer und Wolf der Myelinscheide bei der Bildung der Amyloidkörperchen eine wichtige Rolle zuschreiben. Durch mündliche Mitteilung des Prof. Banti in der Academia MedicoFisica zu Florenz haben wir erfahren, daß dieser Forscher bei Myelinfaserpräparaten Amyloidkörperchen gesehen hat, die mit einem Myelinrand umgeben waren. Wir haben niemals einen solchen Befund gehabt und wir müssen ihn als eine seltene Erscheinung betrachten. Daß Produkte der Degeneration der Markscheide die Bildung der Amyloidkörperchen beeinflussen können, ist sehr möglich, eine wirkliche Zusammenschmelzung beider Elemente ist aber nicht zu sehen. 
Wir haben hier nur von der Form des Vorgangs gesprochen, von seiner Natur und von der chemischen Zusammensetzung der daraus entstandenen Körperchen können wir so gut wie nichts sagen. Es handelt sich hier um einen chronischen Vorgang, der fast regelmäßig beim Alter eintritt, doch nicht ausschließlich, wie man einst glaubte. Karl und Gustav Petren ${ }^{15}$ haben die Körperchen im Rückenmark eines anencephalischen menschlichen Foetus gefunden. Andere Fälle, wo bei jugendlichen Individuen sich Amyloidkörperchen fanden, sind von mehreren Autoren vorgeführt worden. Spiller fand in einem Fall von Landryscher Paralyse zahlreiche Körperchen und zu gleicher Zeit eine hochgradige Arteriosklerose im Zentralnervensystem. Er vermutete einen Zusammenhang zwischen beiden pathologischen Erscheinungen.

Die Körperchen, die Stroebe nach Durchschneidung des Rückenmarks fand, zeigten sich besonders im Gebiete der traumatischen Degeneration. Sehr interessant ist, daß auch bei unvollständiger Trennung des Rückenmarkes im undurchschnittenen Teile Amyloidkörperchen zu sehen waren. Stroebe erklärte ihre Bildung durch den bei der Operation erlittenen Druck und Quetschungen. Wir sind nicht imstande, eine Erklärung für alle diese Fälle zu geben, doch vermuten wir, daß toxische Produkte bei Arteriosklerosen beim Alter und vielleicht anch nach Durchschneidung des Rückenmarks den Vorgang beeinflussen.

Aus unseren Untersuchungen kann man nur schließen, daß die Amyloidkörperchen Degenerationsprodukte der Achsenzylinder sind. Wir sind aber nicht imstande, die Behauptungen der Autoren zu bestreiten, daß die Amyloidkörperchen aus Umwandlungen der Gliazellen entstehen, denn die Amyloidkörperchenbildung könnte eine allgemeinere Form von Degeneration der Elemente im Zentralnervensystem sein. Wir halten dieses aber nicht für wahrscheinlich und jedenfalls die Gründe, die die Autoren für die Annahme der Entstehung aus den Gliazellen, sei es aus den Kernen, wie Redlich, sei es aus dem Protoplasma, wie Obersteiner, angeben, beweisen es nicht. Nur der Nachweis von zahlreichen Übergangsformen könnte zu diesem Schluß berechtigen. Unsere Untersuchungen scheinen 
uns diese Bedingung zu erfüllen und die Phase des Vorganges, wo Amyloidkörperchen in unmittelbarem Zusammenhang mit Achsenzylindern sind, betrachten wir als fundamental für die Erklärung des Prozesses. Daß solche Bilder nur durch Anwendung von Achsenzylinderfärbungsmethoden erreicht werden können, ist verständlich.

\section{Erklärung der Abbildungen auf Taf. XII.}

Fig. 1. Apochrom. Immers. $\frac{1}{1 \frac{2}{2}}$ Koristka. Längsschnitt des Rückenmarks eines Falles von Paralysis agitans. Färbung nach Kaplan. a Polygonales Amyloidkörperchen; b Lücke im Gewebe, die einem Amyloidkörperchen entspricht; c Zusammenschmelzung zweier Amyloidkörperchen. Alle Amyloidkörperchen zeigen eine Spiegelfärbung.

Fig. 2. Apochrom. Immers. $\frac{1}{12}$ Koristka. Längsschnitt des Rückenmarks eines Falles von Paralysis agitans. Färbung nach Kaplan. a Amyloidkörperchen, das mit einem rotgefärbten Achsenzylinder in Zusammenhang steht; eine dünne Kapsel umgibt das Amyloidkörperchen; b Kern einer Gliazelle; c Gliazelle in einer Kette von Amyloidkörperchen und Zellen; d Achsenzylinderanschwellung.

Fig. 3. Apochrom. Immers. $\frac{1}{12}$ Koristka. Längsschnitt der Medulla oblongata eines Falles von seniler Demenz. Silberimprägnation nach Cajal. Die Figur zeigt ein Amyloidkörperchen in deutlichem Zusammenhang mit einem Achsenzylinder.

\section{Literatur.}

1. Purkinje, Bericht über die Naturforscherversammlung zu Prag im Jahre 1837.

2. Virchow, Über eine im Gehirn und im Rückenmark des Menschen aufgefundene Substanz mit der Reaktion der Cellulose. Dieses Archiv, 1854.

3. Luschka, Corpora Amylacea im Ganglion Gasseri. Dieses Archiv, Bd. VI, 1854 .

4. Virchow, Zur Cellulosefrage. Dieses Archiv, 1855.

5. Leber, Handbuch der Augenheilkunde von Graefe und Saemisch, Bd. V, 1875.

6. Treitel, Graefes Archiv für Ophtalmologie, Bd. XXII:

7. Leyden, Zeitschrift für klin. Medizin, Bd. I, 1880.

8. Ceci, Reale Academia dei Lincei, 1881.

9. Homen, Experimenteller Beitrag zur Pathologie und pathol. Anatomie des Rückenmarks, speziell mit Hinsicht auf secundäre Degeneration. Fortschritte der Medizin, 1885. 
10. Schaffer, Pathologie und Anatomie der Lyssa. Zieglers Beiträge, Bd. VII, 1890.

11. Redlich, Die Amyloidkörperchen im Nervensystem. Jahrbücher für Psychiatrie, Bd. X, 1891.

12. Siegert, Untersuchungen über Corpora Amylacea u. Amyloidea. Dieses Archiv, Bd. CXXIX, 1892.

13. Wichmann, Die Amyloiderkrankung. Beiträge zur path. Anat. u. zur allgem. Path., Bd. XIII, 1893.

14. Stroebe, Deg. u. reparat. Vorgänge bei der Heilung der Verletzungen des Rückenmarks. Zieglers Beiträge Bd. XV, 1894.

15. Karl u. Gustav Petren, Beiträge zur Kenntnis des Nervensystems und der Netzhaut bei Anencephalie. Dieses Archiv, Bd. CLI, H. 2, 1898.

16. Spiller, On amyloid, colloid, hyaloid and granular bodies in the central nervons system. New York, Medical Journal, 1898.

17. Fischer, A., Fixierung, Färbung und Bau des Protoplasmas. Jena, G. Fischer, 1899.

18. Obersteiner, Zur Histologie der Gliazellen in den Molekularschichten der Großhirnrinde. Arbeiten aus dem Neurol. Instit. an der Wiener Universität, 1900.

19. Wolf. Die Amyloidkörperchen des Nervensystems. Inaug.-Dissert, München 1901.

20. Kölpin, Tuberkulöse Erkrankung des rechten Atlantoccipitalengelenks. Archiv für Psychiatrie, 1903.

21. Obersteiner, Über das hellgelbe Pigment in den Nervenzellen. Arbeiten aus dem Neurol. Instit. an der Wiener Universität, 1903.

\section{XXIII.}

\section{v. Hansemanns Malakoplakia vesciae urinariae und ihre Beziehungen zur plaqueförmigen Tuberkulose der Harnblase.}

(Aus dem k. k. böhmischen Pathologisch-anatomischen Institute in Prag.) Von Prof, Dr. Rudolf Kimla.

(Mit 2 Figuren im Text.)

I.

Im Jahre 1903 beschrieb v. Han semann ${ }^{1}$ im 173. Bande dieses Archivs eine eigentümliche, von ihm als Malakoplakia bezeichnete Affektion der Harnblase. 